\title{
SyMmetries BETWEEN TWO RAMSEY PROPERTIES
}

\author{
Lorenz Halbeisen円 \\ Université de Caen \\ France
}

\begin{abstract}
In this article we compare the well-known Ramsey property with a dual form of it, the so called dual-Ramsey property (which was suggested first by Carlson and Simpson). Even if the two properties are different, it can be shown that all classical results known for the Ramsey property also hold for the dual-Ramsey property. We will also show that the dual-Ramsey property is closed under a generalized Suslin operation (the similar result for the Ramsey property was proved by Matet). Further we compare two notions of forcing, the Mathias forcing and a dual form of it, and will give some symmetries between them. Finally we give some relationships between the dual-Mathias forcing and the dual-Ramsey property.
\end{abstract}

\section{Notations and definitions}

Most of our set-theoretic notations and notations of forcings are standard and can be found in Je 1] or $\mathrm{Ku}$. An exception is that we will write $A^{B}$ for the set of all functions from $B$ to $A$, instead of $B_{A}$ because we never use ordinal arithmetic. $A^{<\omega}$ is the set of all partial functions $f$ from $\omega$ to $A$ such that the cardinality of $\operatorname{dom}(f)$ is finite.

First we will give the definitions of the sets we will consider as the real numbers. Let $[x]^{\kappa}:=\{y \subseteq x:|y|=\kappa\}$ and $[x]^{<\kappa}:=\{y \subseteq x:|y|<\kappa\}$, where $|y|$ denotes the cardinality of $y$. For $x \in[\omega]^{\omega}$, we will consider $[x]^{<\omega}$ as the set of strictly increasing, finite sequences in $x$ and $[x]^{\omega}$ as the set of strictly increasing, infinite sequences in $x$. For $x \in[\omega]^{\omega}$ and $n \in \omega$ let $x(n)$ be such that $x(n) \in x$ and $|x(n) \cap x|=n$.

We can consider $[\omega]^{\omega}$ also as the set of infinite 0 -1-sequences (denoted by $2^{\omega}$ ) or as the set of all infinite sequences in $\omega$ (denoted by $\omega^{\omega}$ ).

\section{The Ellentuck topology}

We define a topology on $[\omega]^{\omega}$. Let $X \in[\omega]^{\omega}$ and $s \in[\omega]^{<\omega}$ such that $\max (s)<$ $\min (X)$; then $[s, X]^{\omega}:=\left\{Y \in[\omega]^{\omega}: Y \subseteq(s \cup X) \wedge s \subseteq Y\right\}$. Now let the basic open sets on $[\omega]^{\omega}$ be the sets $[s, X]^{\omega}$. These sets are called the Ellentuck neighborhoods. The topology induced by the Ellentuck neighborhoods is called the Ellentuck topology.

\footnotetext{
${ }^{1}$ The author wishes to thank the Swiss National Science Foundation for supporting him.
} 


\section{Relations on the set of partitions}

A partition $X$ (of $\omega$ ) is a subset of $\mathcal{P}(\omega)$ such that the following holds:

i) if $b \in X$ then $b \neq \emptyset$

ii) if $b_{1}, b_{2} \in X$ and $b_{1} \neq b_{2}$ then $b_{1} \cap b_{2}=\emptyset$

iii) $\bigcup X=\omega$.

A partition means always a partition of $\omega$. If $X$ is a partition and $b \in X$ then we call $b$ a block of $X$. If a partition has infinitely many blocks (or equivalently if $X$ is infinite) we call $X$ an infinite partition. The set of all infinite partitions is denoted by $(\omega)^{\omega}$.

If $X$ is a partition, $b \in X$ and $n, m \in \omega$ both belong to $b$, then we write $\natural_{X}(n, m)$. On the other hand with $\left\{\{n, m\} \in[\omega]^{2}: \bigsqcup_{X}(n, m)\right\}$ we can reconstruct the partition $X$.

A partial partition $X^{\prime}$ is a subset of $\mathcal{P}(\omega)$ such that (i) and (ii) hold but instead of (iii) we have

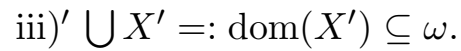

Note that a partition is always also a partial partition. If $\operatorname{dom}\left(X^{\prime}\right) \in \omega$ then $X^{\prime}$ is a partition of some $n \in \omega$. The set of all partial partitions $X^{\prime}$ where $\operatorname{dom}\left(X^{\prime}\right) \in \omega$ is denoted by $(\mathbb{N})$. For $s \in(\mathbb{I N}), s^{*}$ denotes the partial partition $s \cup\{\{\operatorname{dom}(s)\}\}$.

Let $X_{1}, X_{2}$ be two partial partitions. We say that $X_{1}$ is coarser than $X_{2}$, or that $X_{2}$ is finer than $X_{1}$, and write $X_{1} \sqsubseteq X_{2}$ if for all blocks $b \in X_{1}$ the set $b \cap \operatorname{dom}\left(X_{2}\right)$ is the union of some sets $b_{i} \cap \operatorname{dom}\left(X_{1}\right)$, where each $b_{i}$ is a block of $X_{2}$. Let $X_{1} \sqcap X_{2}$ denote the finest partial partition which is coarser than $X_{1}$ and $X_{2}$ such that $\operatorname{dom}\left(X_{1} \sqcap X_{2}\right)=\operatorname{dom}\left(X_{1}\right) \cup \operatorname{dom}\left(X_{2}\right)$.

If $f \in[\omega]^{<\omega}$ is a finite subset of $\omega$, then $\{f\}$ is a partial partition with $\operatorname{dom}(\{f\})=f$. For two partial partitions $X_{1}$ and $X_{2}$ we write $X_{1} \sqsubseteq^{*} X_{2}$ if there is a finite set $f \subseteq \operatorname{dom}\left(X_{1}\right)$ such that $X_{1} \sqcap\{f\} \sqsubseteq X_{2}$ and say that $X_{1}$ is coarser* than $X_{2}$. If $X_{1} \sqsubseteq^{*} X_{2}, X_{2} \sqsubseteq^{*} X_{1}$ and $\operatorname{dom}\left(X_{1}\right)=\operatorname{dom}\left(X_{2}\right)$, then we write $X_{1} \stackrel{*}{=} X_{2}$.

Let $X_{1}, X_{2}$ be two partial partitions. If each block of $X_{1}$ can be written as the intersection of a block of $X_{2}$ with $\operatorname{dom}\left(X_{1}\right)$, then we write $X_{1} \preceq X_{2}$. Note that $X_{1} \preceq X_{2}$ implies $\operatorname{dom}\left(X_{1}\right) \subseteq \operatorname{dom}\left(X_{2}\right)$.

If $X$ is a partial partition, then $\operatorname{Min}(X)$ denotes the set $\{n \in \omega: \exists b \in X(n=$ $\min (b))\}$, where $\min (b):=\bigcap b$. If we order the blocks of $X$ by their least element, then $X(n)$ denotes the $n$th block in this ordering and $X(n)(k)$ denotes the $k$ th element (in the natural ordering) belonging to this block.

\section{The dual Ellentuck topology}

We define a topology on the set of partitions as follows. Let $X \in(\omega)^{\omega}$ and $s \in(\mathbb{N})$ such that $s \sqsubseteq X$. Then $(s, X)^{\omega}:=\left\{Y \in(\omega)^{\omega}: s \preceq Y \wedge Y \sqsubseteq X\right\}$ and $(X)^{\omega}:=(\emptyset, X)^{\omega}$. Now let the basic open sets on $(\omega)^{\omega}$ be the sets $(s, X)^{\omega}$ (where $X$ and $s$ as above). These sets are called the dual Ellentuck neighborhoods. 
The topology induced by the dual Ellentuck neighborhoods is called the dual Ellentuck topology (cf. CS).

\section{Two notions of forcing}

The Mathias forcing $\mathbf{M}$ is defined as follows:

$$
\begin{aligned}
& \langle s, S\rangle \in \mathbf{M} \Leftrightarrow s \in[\omega]^{<\omega} \wedge S \in[\omega]^{\omega} \wedge \max (s)<\min (S), \\
& \langle s, S\rangle \leq\langle t, T\rangle \Leftrightarrow t \subseteq s \wedge S \subseteq T \wedge \forall n \in(s \backslash t)(n \in T) .
\end{aligned}
$$

If $\langle s, S\rangle$ is an M-condition, then we call $s$ the stem of the condition. The Mathias forcing $\mathbf{M}$ has a lot of combinatorial properties (which can be found in [Ma and [JS] or in [H]]). Note that we can consider an M-condition $\langle s, S\rangle$ as an Ellentuck neighborhood $[s, S]^{\omega}$ and $\langle s, S\rangle \leq\langle t, T\rangle$ if and only if $[s, S]^{\omega} \subseteq[t, T]^{\omega}$. The dual-Mathias forcing $\mathfrak{M}$ is defined similarly to the Mathias forcing $\mathbf{M}$, using the dual Ellentuck topology instead of the Ellentuck topology. So,

$$
\langle s, X\rangle \in \mathfrak{M} \Leftrightarrow(s, X)^{\omega} \text { is a dual Ellentuck neighborhood }
$$

and

$$
\langle s, X\rangle \leq\langle t, Y\rangle \Leftrightarrow(s, X)^{\omega} \subseteq(t, Y)^{\omega} .
$$

If $\langle s, X\rangle$ is an $\mathfrak{M}$-condition, then we call $s$ again the stem of the condition. Because the dual-Mathias forcing is very close to the usual Mathias forcing, it also has some nice properties similar to those of $\mathbf{M}$.

\section{Two Ramsey properties}

The classical Ramsey property is a property of sets of infinite subsets of $\omega$ (of sets of reals). A set $A \subseteq[\omega]^{\omega}$ has the Ramsey property (or is Ramsey) if $\exists X \in[\omega]^{\omega}\left([X]^{\omega} \subseteq A \vee[X]^{\omega} \cap A=\emptyset\right)$. If there exists an $X$ such that $[X]^{\omega} \cap A=\emptyset$ we call $A$ a Ramsey null set. A set $A \subseteq[\omega]^{\omega}$ is completely Ramsey if for every Ellentuck neighborhood $[s, Y]^{\omega}$ there is an $X \in[s, Y]^{\omega}$ such that $[s, X]^{\omega} \subseteq A$ or $[s, X]^{\omega} \cap A=\emptyset$. If we are always in the latter case, then we call $A$ completely Ramsey null.

The dual-Ramsey property deals with sets of infinite partitions of $\omega$. A set $A \subseteq$ $(\omega)^{\omega}$ has the dual-Ramsey property (or is dual-Ramsey) if $\exists X \in(\omega)^{\omega}\left((X)^{\omega} \subseteq\right.$ $\left.A \vee(X)^{\omega} \cap A=\emptyset\right)$. If there exists an $X$ such that $(X)^{\omega} \cap A=\emptyset$ we call $A$ a dualRamsey null set. A set $A \subseteq(\omega)^{\omega}$ is completely dual-Ramsey if for every dual Ellentuck neighborhood $(s, Y)^{\omega}$ there is an $X \in(s, Y)^{\omega}$ such that $(s, X)^{\omega} \subseteq A$ or $(s, X)^{\omega} \cap A=\emptyset$. If we are always in the latter case, then we call $A$ completely dual-Ramsey null.

Now we can start to give some symmetries between the two Ramsey properties and between the two Mathias forcings.

\section{Basic facts}

In this section we give the tools to consider sets of partitions as sets of reals and to compare the two Ramsey properties. We will give also some basic facts 
and well-known results concerning the dual-Ramsey property and dual-Mathias forcing. Further we give some symmetries between Mathias forcing and the dual-Mathias forcing.

To compare the two Ramsey properties we first show that we can consider each $A \subseteq[\omega]^{\omega}$ as a set of infinite partitions of $\omega$ and vice versa. For this we define some arithmetical relations and functions.

Let $n, m \in \omega$ then $\operatorname{div}(n, m):=\max \left(\{k \in \omega: k \cdot m \leq n\}\right.$. For $\{n, m\} \in[\omega]^{2}$ let $b\{n, m\}:=\frac{1}{2}\left(\max (\{n, m\})^{2}-\max (\{n, m\})\right)+\min (\{n, m\})$. Consider $b\{n, m\}$ as undefined for $n=m$.

Let $x \in[\omega]^{\omega}$; then $\operatorname{trans}(x) \subseteq \omega$ is such that $n \notin \operatorname{trans}(x)$ iff there is a finite sequence $s$ of natural numbers of length $l+1$ such that

$$
n=b\{s(0), s(l)\} \text { and } \forall k \in\{1, \ldots, l\}(b\{s(k-1), s(k)\} \notin x) .
$$

Note that $\operatorname{trans}(x) \subseteq x$. If $x \in[\omega]^{\omega}$, then we can consider $x$ as a partition with

$$
\natural_{x}(n, m) \text { if and only if } n=m \text { or } b\{n, m\} \notin \operatorname{trans}(x) \text {. }
$$

The corresponding partition of a real $x \in[\omega]^{\omega}$ is denoted by $\operatorname{cp}(x)$. Note that $\operatorname{cp}(x) \in(\omega)^{\omega}$ iff $\forall k \exists n>k \forall m<n\left(\neg \natural_{x}(n, m)\right)$ and further if $y \subseteq x$, then $\mathrm{cp}(y) \sqsubseteq \mathrm{cp}(x)$.

A partition $X$ of $\omega$ we encode by a real $\mathrm{pc}(X)$ (the partition code of $X$ ) as follows.

$$
\operatorname{pc}(X):=\left\{k \in \omega: \exists n m\left(k=b\{n, m\} \wedge \neg \natural_{X}(n, m)\right\} .\right.
$$

Note that if $X_{1} \sqsubseteq X_{2}$ then $\operatorname{pc}\left(X_{1}\right) \subseteq \operatorname{pc}\left(X_{2}\right)$. With these definitions we get the

FACT 2.1 The dual Ellentuck topology is finer than the topology of the Baire space.

Proof: Let $s \in \omega^{<\omega}$ and $U_{s}=\left\{f \in \omega^{\omega}: s \subset f\right\}$ be a basic open set in the Baire space $\omega^{\omega}$. Because there is a bijection between $\omega^{\omega}$ and $[\omega]^{\omega}$, we can write $U_{s}$ as a set $V_{s^{\prime}}=\left\{r \in[\omega]^{\omega}: s^{\prime} \subset r \wedge \min (r \backslash s)>\max (s)\right\}$. Now $\operatorname{cp}\left[V_{s^{\prime}}\right] \cap(\omega)^{\omega}$ (where $\mathrm{cp}\left[V_{s^{\prime}}\right]:=\left\{\mathrm{cp}(r): r \in V_{s^{\prime}}\right\}$ ) is open with respect to the dual Ellentuck topology. Therefore the dual Ellentuck topology is finer than the topology of the Baire space. $\dashv$

REMARK: A similar result is true for the Ellentuck topology (cf. [E]).

FACT 2.2 A set $C \subseteq(\omega)^{\omega}$ is completely dual-Ramsey if and only if $C$ has the Baire property with respect to the dual Ellentuck topology and it is completely dual-Ramsey null if and only if it is meager with respect to the dual Ellentuck topology.

PROOF: This is proved in CS. $\dashv$

REMARK: The analogous result is known for the Ramsey property with respect to the Ellentuck topology (cf. E[]). 


\section{Some symmetries between the two Mathias forcings}

If $\mathfrak{g}$ is $\mathfrak{M}$-generic over $V$ and $\mathfrak{g}^{\prime} \in(\mathfrak{g})^{\omega}$, then also $\mathfrak{g}^{\prime}$ is $\mathfrak{M}$-generic over $V$ (cf. [CS] Theorem 5.5). From this it follows immediately that $\mathfrak{M}$ is proper and therefore does not collapse $\aleph_{1}$. (For the definition of properness consider e.g. Go.)

Further, for any $\mathfrak{M}$-condition $\langle s, X\rangle$ and any sentence $\Phi$ of the forcing language $\mathfrak{M}$ there is an $\mathfrak{M}$-condition $\langle s, Y\rangle \leq\langle s, X\rangle$ such that $\langle s, Y\rangle \Vdash_{\mathfrak{M}} \Phi$ or $\langle s, Y\rangle \Vdash \mathfrak{M} \neg \Phi$ (cf. [CS] Theorem 5.2). This property is called pure decision.

REMARK: The similar results for Mathias forcing $\mathbf{M}$ can be found in Ma (or in Je 2]).

We can write the dual-Mathias forcing as a two step iteration where the first is the forcing notion $\mathfrak{U}$.

Let $\mathfrak{U}$ be the partial order defined as follows:

$$
\begin{aligned}
p \in \mathfrak{U} & \Leftrightarrow p \in(\omega)^{\omega}, \\
p \leq q & \Leftrightarrow p \sqsubseteq^{*} q .
\end{aligned}
$$

We can also write the Mathias forcing as a two step iteration, where the first step is the forcing notion $\mathbf{U}$. Let $\mathcal{J}:=[\omega]^{<\omega}$ be the ideal of finite sets and let $\left\langle[\omega]^{\omega} / \mathcal{J}, \leq\right\rangle=: \mathbf{U}$ be the partial order defined as follows. $p \in \mathbf{U} \Leftrightarrow p \in[\omega]^{\omega}$ and $p \leq q \Leftrightarrow p \backslash q \in \mathcal{J}$ (this is $p \subseteq^{*} q$ ).

FACT 2.3 The forcing notion $\mathfrak{U}$ is $\aleph_{0}$-closed and if $\mathfrak{D}$ is $\mathfrak{U}$-generic over $V$, then $\operatorname{Min}(\mathfrak{D})$ is a Ramsey ultrafilter in $V[\mathfrak{D}]$.

Proof: Let $X_{1} \geq X_{2} \geq \ldots$ be a decreasing sequence in $\mathfrak{U}$. Choose a sequence $f_{i}(i \in \omega)$ of finite sets of natural numbers, such that $X_{i+1} \sqcap\left\{f_{i}\right\} \sqsubseteq X_{i}$. Define $y_{0}:=X_{0}(0)$ and $y_{n}:=X_{n}(k)$ where $k:=3+\bigcup_{i<n}\left(\bigcup f_{i}\right)$. Now $Y:=\left\{y_{i}: i \in\right.$ $\omega\} \cup\left(\omega \backslash \bigcup_{i \in \omega} y_{i}\right)$ is coarser* than each $X_{i}(i \in \omega)$ and therefore $\mathfrak{U}$ is $\aleph_{0}$-closed. Now we claim that the set $\{\operatorname{Min}(X): X \in \mathfrak{D}\}$ is a Ramsey ultrafilter in $V[\mathfrak{D}]$. Remember that a forcing notion which is $\aleph_{0}$-closed adds no new reals to $V$ (cf. Je 1 Lemma 19.6). Take a $\pi \in 2^{[\omega]^{2}}$ and a $Y \in(\omega)^{\omega}$; then by the Ramsey Theorem (cf. Je 1] Lemma 29.1) for $\operatorname{Min}(Y) \in[\omega]^{\omega}$ there exists an infinite $r \subseteq \operatorname{Min}(Y)$ such that $\pi$ is constant on $[r]^{2}$. Now let $X:=\{b: b \in$ $Y \wedge b \cap r \neq \emptyset\} \cup \bigcup\{b: b \in Y \wedge b \cap r=\emptyset\}$; then $X \sqsubseteq Y$ and $\operatorname{Min}(X)=r$. Thus $H_{\pi}:=\left\{X \in(\omega)^{\omega}:\left.\pi\right|_{[\operatorname{Min}(X)]^{2}}\right.$ is constant $\}$ is dense in $\mathfrak{U}$ and hence $H_{\pi} \cap \mathfrak{D} \neq \emptyset$. $\dashv$

REMARK: It is easy to see that the forcing notion $\mathbf{U}$ is $\aleph_{0}$-closed. Further we have that if $D$ is $\mathbf{U}$-generic over $V$, then $D$ is a Ramsey ultrafilter in $V[D]$. The forcing notion $\mathfrak{U}$ is stronger than the forcing notion $\mathbf{U}$.

FACT 2.4 If $\mathfrak{D}$ is $\mathfrak{U}$-generic, then the set $\{\operatorname{Min}(X): X \in \mathfrak{D}\}$ is $\mathbf{U}$-generic.

Proof: Let $A \subseteq[\omega]^{\omega}$ be a maximal anti-chain in $\mathbf{U}$, i.e., $A$ is a maximal almost disjoint family. Then the set $D_{A}:=\left\{X \in \mathfrak{U}: \exists a \in A\left(\operatorname{Min}(X) \subseteq^{*} a\right)\right\}$ is dense in $\mathfrak{U}$. $\dashv$

We define now the second step of the two step iteration.

Let $\mathfrak{F} \subseteq(\omega)^{\omega}$. The partial order $\mathbf{P}_{\mathfrak{F}}$ is defined as follows. 
$\langle s, X\rangle \in \mathbf{P}_{\mathfrak{F}} \Leftrightarrow s \in(\mathbb{N}) \wedge X \in \mathfrak{F} \wedge(s, X)^{\omega}$ is a dual Ellentuck neighborhood, $\langle s, X\rangle \leq\langle t, Y\rangle \Leftrightarrow(s, X)^{\omega} \subseteq(t, Y)^{\omega}$.

REMARK: For $\mathcal{F} \subseteq[\omega]^{\omega}$ we can define the partial order $\mathbf{P}_{\mathcal{F}}$ similarly.

FACT 2.5 Let $\tilde{\mathfrak{D}}$ be the canonical $\mathfrak{U}$-name for the $\mathfrak{U}$-generic object; then

$$
\mathfrak{U} * \mathbf{P}_{\tilde{\mathfrak{D}}} \approx \mathfrak{M}
$$

\section{ProOF:}

$$
\begin{aligned}
\mathfrak{U} * \mathbf{P}_{\tilde{\mathfrak{D}}} & =\left\{\langle p,\langle\tilde{s}, \tilde{X}\rangle\rangle: p \in \mathfrak{U} \wedge p \Vdash \mathfrak{U}\langle\tilde{s}, \tilde{X}\rangle \in \mathbf{P}_{\tilde{\mathfrak{D}}}\right\} \\
& =\left\{\langle p,\langle\tilde{s}, \tilde{X}\rangle\rangle: p \in(\omega)^{\omega} \wedge p \Vdash \mathfrak{U}(\tilde{X} \in \tilde{\mathfrak{D}} \wedge \tilde{s} \sqsubseteq \tilde{X})\right\} .
\end{aligned}
$$

Now the embedding

$$
\begin{array}{cccc}
h: & \mathfrak{M} & \longrightarrow & \mathfrak{U} * \mathbf{P}_{\tilde{\mathfrak{D}}} \\
& \langle s, X\rangle & \longmapsto & \langle X,\langle\check{s}, \tilde{X}\rangle\rangle
\end{array}
$$

is a dense embedding (see G0] Definition 0.8):

1. It is easy to see that $h$ preserves the order relation $\leq$.

2. Let $\langle p,\langle\tilde{s}, \tilde{X}\rangle\rangle \in \mathfrak{U} * \mathbf{P}_{\tilde{\mathfrak{D}}}$. Because $\mathfrak{U}$ is $\aleph_{0}$-closed, there is a condition $q \leq p$ and $s \in(\mathbb{N}), X \in(\omega)^{\omega}$ such that $q \Vdash \mathfrak{U} \check{\mathfrak{s}}=\tilde{s} \wedge \check{X}=\tilde{X}$. Evidently, $\langle q,\langle\check{s}, \check{X}\rangle\rangle \in \mathfrak{U} * \mathbf{P}_{\tilde{\mathfrak{D}}}$ is stronger than $\langle p,\langle\tilde{s}, \tilde{X}\rangle\rangle$. Let $Z:=q \sqcap X$ and let $Z^{\prime} \sqsubseteq^{*} Z$ be such that $s \sqsubseteq Z^{\prime}$. Now we have $h\left(\left\langle s, Z^{\prime}\right\rangle\right) \leq\langle p,\langle\tilde{s}, \tilde{X}\rangle\rangle . \dashv$

REMARK: Let $\tilde{D}$ be the canonical U-name for the U-generic object, then $\mathbf{U} * \mathbf{P}_{\tilde{D}} \approx \mathbf{M}$.

The dual-Mathias forcing is stronger than the Mathias forcing.

FACT 2.6 The dual-Mathias forcing adds Mathias reals.

Proof: Let $\mathfrak{D}$ be $\mathfrak{U}$-generic over $V$; then by Fact 2.4, $D:=\{\operatorname{Min}(X): X \in$ $\mathfrak{D}\}$ is $\mathbf{U}$-generic over $V$. Now we define $h: \mathbf{P}_{\mathfrak{D}} \rightarrow \mathbf{P}_{D}$ as follows.

$$
\begin{aligned}
h: \quad \mathbf{P}_{\mathfrak{D}} & \longrightarrow \mathbf{P}_{D} \\
\langle s, X\rangle & \longmapsto
\end{aligned}
$$

For $h$ the following is true.

(i) If $q_{1}, q_{2} \in \mathbf{P}_{\mathfrak{D}}, q_{1} \leq q_{2}$, then $h\left(q_{1}\right) \leq h\left(q_{2}\right)$.

(ii) $\forall q \in \mathbf{P}_{\mathfrak{D}} \forall p^{\prime} \leq h(q) \exists q^{\prime} \in \mathbf{P}_{\mathfrak{D}}$ such that $q$ and $q^{\prime}$ are compatible and $h\left(q^{\prime}\right) \leq$ $p^{\prime}$.

Therefore with Je 2 Part I, Lemma 2.7 we finally get $V^{\mathbf{M}} \subseteq V^{\mathfrak{M}}$. 


\section{On the dual-Ramsey property}

In this section we will show that the dual-Ramsey property is closed under a generalized Suslin operation. As a corollary we will get the already known result that analytic sets are completely dual-Ramsey.

Let $\mathfrak{J} \subseteq \mathcal{P}\left((\omega)^{\omega}\right)$ be the set of all completely dual-Ramsey null sets. Further let $\operatorname{add}(\mathfrak{J})$ be the smallest cardinal $\kappa$ such that there exists a family $\mathcal{F}=\left\{J_{\alpha} \in \mathfrak{J}\right.$ : $\alpha<\kappa\}$ with $\bigcup \mathcal{F} \notin \mathfrak{J}$ and let $\operatorname{cov}(\mathfrak{J})$ be the smallest cardinal $\kappa$ such that there exists a family $\mathcal{F}=\left\{J_{\alpha} \in \mathfrak{J}: \alpha<\kappa\right\}$ with $\bigcup \mathcal{F}=(\omega)^{\omega}$. In Ha it is shown that $\operatorname{cov}(\mathfrak{J})=\operatorname{add}(\mathfrak{J})=\mathfrak{H}$, where $\mathfrak{H}$ is the dual-shattering cardinal. Further it is shown that $\mathfrak{H}>\omega_{1}$ is relatively consistent with ZFC.

Let $\operatorname{Seq}(\kappa):=\kappa^{<\omega}$ and for $f \in \kappa^{\omega}, n \in \omega$, let $\bar{f}(n)$ denote the finite sequence $\langle f(0), f(1), \ldots, f(n-1)\rangle$. The generalized Suslin operation $\mathcal{A}_{\kappa}$ (for a cardinal $\kappa)$ is defined as follows:

$$
\mathcal{A}_{\kappa}\left\{Q_{s}: s \in \operatorname{Seq}(\kappa)\right\}:=\bigcup_{f \in \kappa^{\omega}} \bigcap_{n \in \omega} Q_{\bar{f}(n)} .
$$

In Theorem 3.5 below we will show that for each cardinal $\kappa<\mathfrak{H}$, the completely dual-Ramsey sets are closed under the operation $\mathcal{A}_{\kappa}$. But first we give some other results.

A set $R \subseteq(\omega)^{\omega}$ is dual Ellentuck meager if $R$ is meager with respect to the dual Ellentuck topology. Remember that a set is dual Ellentuck meager if and only if it is completely dual-Ramsey null and a set is completely dual-Ramsey if and only if it has the Baire property with respect to the dual Ellentuck topology.

If $(s, X)^{\omega}$ is a dual Ellentuck neighborhood then we say that $R$ is dual Ellentuck meager in $(s, X)^{\omega}$ if $R \cap(s, X)^{\omega}$ is dual Ellentuck meager. By [CS] Theorem 4.1, $R$ is dual Ellentuck meager in $(s, X)^{\omega}$ if for all $(t, Y)^{\omega} \subseteq(s, X)^{\omega}$ there exists a partition $Z \in(t, Y)^{\omega}$ such that $(t, Z)^{\omega} \cap R=\emptyset$.

Let $R \subseteq(\omega)^{\omega}$ and $M:=\bigcup\left\{(s, X)^{\omega}: R\right.$ is dual Ellentuck meager in $\left.(s, X)^{\omega}\right\}$. Further let $M(R):=M \cap R$. We first show that

LEMMA 3.1 If $(s, X)^{\omega}$ is a dual Ellentuck neighborhood such that $(s, X)^{\omega} \subseteq M$, then $R$ is dual Ellentuck meager in $(s, X)^{\omega}$.

Proof: If $(s, X)^{\omega} \subseteq M$, then $(s, X)^{\omega}=\bigcup\left\{(t, Y)^{\omega} \subseteq(s, X)^{\omega}: R\right.$ is dual Ellentuck meager in $\left.(t, Y)^{\omega}\right\}$. Let $N:=\bigcup\left\{(u, Z)^{\omega} \subseteq(s, X)^{\omega}: R \cap(u, Z)^{\omega}=\emptyset\right\}$. Because $N$ is an open set, $N$ is completely dual-Ramsey. Therefore, for any $(t, Y)^{\omega} \subseteq(s, X)^{\omega}$ there exists a partition $Y^{\prime} \in(t, Y)^{\omega}$ such that $\left(t, Y^{\prime}\right)^{\omega} \subseteq N$ or $\left(t, Y^{\prime}\right)^{\omega} \cap N=\emptyset$. If we are in the latter case, then because $\left(t, Y^{\prime}\right)^{\omega} \subseteq(s, X)^{\omega}$, we find a $\left(u, Y^{\prime \prime}\right)^{\omega} \subseteq\left(t, Y^{\prime}\right)^{\omega}$ such that $R$ is dual Ellentuck meager in $\left(u, Y^{\prime \prime}\right)^{\omega}$. Hence, there exists a $(u, Z)^{\omega} \subseteq\left(u, Y^{\prime \prime}\right)^{\omega}$ such that $(u, Z)^{\omega} \cap R=\emptyset$, which contradicts $\left(t, Y^{\prime}\right)^{\omega} \cap N=\emptyset$. So we are always in the former case, which implies that $R$ is dual Ellentuck meager in $(s, X)^{\omega}$.

With this result, we can easily prove the following

LEMMA 3.2 The set $M(R)$ is dual Ellentuck meager. 
Proof: Take a dual Ellentuck neighborhood $(s, X)^{\omega}$ and let $S:=\bigcup\left\{(t, Z)^{\omega} \subseteq\right.$ $(s, X)^{\omega}: R$ is dual Ellentuck meager in $\left.(t, Z)^{\omega}\right\}$. Then $S$ as the union of open sets is open and a subset of $(s, X)^{\omega}$. Because $(s, X)^{\omega}$ is also closed (in the dual Ellentuck topology), the set $C:=(s, X)^{\omega} \backslash S$ is closed. By CS Theorem 4.1 the sets $C$ and $S$ both are completely dual-Ramsey. Therefore we find for every $\left(s^{\prime}, X^{\prime}\right)^{\omega} \subseteq(s, X)^{\omega}$ a partition $Y \in\left(s^{\prime}, X^{\prime}\right)^{\omega}$ such that $\left(s^{\prime}, Y\right)^{\omega} \subseteq S$ or $\left(s^{\prime}, Y\right)^{\omega} \subseteq C$. Now if $\left(s^{\prime}, Y\right)^{\omega} \subseteq S$, then by Lemma 3.1, $R$ is dual Ellentuck meager in $\left(s^{\prime}, Y\right)^{\omega}$ and if $\left(s^{\prime}, Y\right)^{\omega} \subseteq C$, then $\left(s^{\prime}, Y\right)^{\omega} \cap M(R)=\emptyset$. To see this, assume there is an $H \in M(R) \cap\left(s^{\prime}, Y\right)^{\omega}$. Because $H \in M(R)$ there exists a dual Ellentuck neighborhood $(t, Z)^{\omega}$ such that $H \in(t, Z)^{\omega}$ and $R$ is dual Ellentuck meager in $(t, Z)^{\omega}$. Because $H \in(t, Z)^{\omega}$ and $H \in\left(s^{\prime}, Y\right)^{\omega}$ there is a dual Ellentuck neighborhood $(u, U)^{\omega} \subseteq(t, Z)^{\omega} \cap\left(s^{\prime}, Y\right)^{\omega}$. But with $(u, U)^{\omega} \subseteq(t, Z)^{\omega}$ it follows that $R$ is dual Ellentuck meager in $(u, U)^{\omega}$ and therefore $(u, U)^{\omega} \subseteq S$, a contradiction to $(u, U)^{\omega} \subseteq\left(s^{\prime}, Y\right)^{\omega} \subseteq C$.

Therefore, in both cases $M(R)$ is dual Ellentuck meager in $\left(s^{\prime}, Y\right)^{\omega} \subseteq\left(s^{\prime}, X^{\prime}\right)^{\omega}$ and because $(s, X)^{\omega}$ and $\left(s^{\prime}, X^{\prime}\right)^{\omega} \subseteq(s, X)^{\omega}$ were arbitrary, the set $M(R)$ is dual Ellentuck meager in each dual Ellentuck neighborhood. Hence, the set $M(R)$ is dual Ellentuck meager.

COROLLARY 3.3 The set $R \cup\left((\omega)^{\omega} \backslash M\right)$ has the dual Ellentuck Baire property.

Proof: Because $M$ is open, $(\omega)^{\omega} \backslash M$ is closed and $R \cup\left((\omega)^{\omega} \backslash M\right)=(R \cap$ $M) \cup\left((\omega)^{\omega} \backslash M\right)=M(R) \cup\left((\omega)^{\omega} \backslash M\right)$ which is the union of a meager set and a closed set and therefore has the dual Ellentuck Baire property.

THEOREM 3.4 If $R \subseteq(\omega)^{\omega}$, then we can construct a set $A \supseteq R$ which has the dual Ellentuck Baire property and whenever $Z \subseteq A \backslash R$ has the dual Ellentuck Baire property, then $Z$ is dual Ellentuck meager.

Proof: Let $A:=R \cup\left((\omega)^{\omega} \backslash M\right)$ where $M:=\bigcup\left\{(s, X)^{\omega}: R\right.$ is dual Ellentuck meager in $\left.(s, X)^{\omega}\right\}$. By Lemma 3.2 and Corollary 3.3 we know that $A$ has the dual Ellentuck Baire property. Now let $Z \subseteq A \backslash R$ with the dual Ellentuck Baire property. If $Z$ is not dual Ellentuck meager, then there exists a dual Ellentuck neighborhood $(u, U)^{\omega}$, such that $(u, U)^{\omega} \backslash Z$ and therefore $(u, U)^{\omega} \cap R$ are dual Ellentuck meager. Hence, $R$ is dual Ellentuck meager in $(u, U)^{\omega}$ and therefore $(u, U)^{\omega} \subseteq M$. Since $(u, U)^{\omega} \cap Z \neq \emptyset$ and $Z \cap M=\emptyset$, there is a $Y \in(u, U)^{\omega}$ such that $Y \notin M$, a contradiction to $R$ is dual Ellentuck meager in $(u, U)^{\omega}$.

Now we can prove the following

THEOREM 3.5 Let $\kappa<\mathfrak{H}$ be a cardinal number and for each $s \in \operatorname{Seq}(\kappa)$ let $Q_{s} \subseteq(\omega)^{\omega}$. If all the sets $Q_{s}$ are completely dual-Ramsey, then the set

$$
\mathcal{A}_{\kappa}\left\{Q_{s}: s \in \operatorname{Seq}(\kappa)\right\}
$$

is completely dual-Ramsey too.

Proof: Let $\left\{Q_{s}: s \in \operatorname{Seq}(\kappa)\right\}$ be a set of completely dual-Ramsey sets and let $A:=\mathcal{A}_{\kappa}\left\{Q_{s}: s \in \operatorname{Seq}(\kappa)\right\}$. For two sequences $s, f \in \kappa^{\leq \omega}$ we write $s \subseteq f$ if 
$s$ is an initial segment of $f$. If $s \in \kappa^{<\omega}$ is a finite sequence, then $|s|$ denotes the length of $s$. Without loss of generality we may assume that $Q_{s} \supseteq Q_{t}$ whenever $s \subseteq t$.

For $s \in \operatorname{Seq}(\kappa)$ let

$$
A_{s}:=\bigcup_{\substack{f \in \kappa^{\omega} \\ s \subseteq f}} \bigcap_{\substack{n \in \omega \\ n \geq|s|}} Q_{\bar{f}(n)} .
$$

For $s \in \operatorname{Seq}(\kappa)$ we have $A_{s} \subseteq Q_{s}, A_{s}=\bigcup_{\alpha<\kappa} A_{s-\alpha}$ and $A=A_{\emptyset}$. By Theorem 3.4, for each $s \in \operatorname{Seq}(\kappa)$ we find a $B_{s} \supseteq A_{s}$ which is completely dual-Ramsey and if $Z \subseteq B_{s} \backslash A_{s}$ has the dual-Ramsey property, then $Z$ is dual-Ramsey null. Because $Q_{s} \supseteq A_{s}$ is completely dual-Ramsey, we may assume that $B_{s} \subseteq Q_{s}$ and therefore

$$
A=\mathcal{A}_{\kappa}\left\{B_{s}: s \in \operatorname{Seq}(\kappa)\right\} .
$$

Let $B:=B_{\emptyset}$. Note that $A=\bigcup_{\alpha<\kappa} A_{\langle\alpha\rangle} \subseteq \bigcup_{\alpha<\kappa} B_{\langle\alpha\rangle}$ and therefore $B \subseteq$ $\bigcup_{\alpha<\kappa} B_{\langle\alpha\rangle}$. Now we show that

$$
B \backslash A \subseteq \bigcup_{\alpha<\kappa} B_{\langle\alpha\rangle} \subseteq \bigcup_{f \in \kappa^{\omega}} \bigcap_{n \in \omega} B_{\bar{f}(n)} \subseteq \bigcup_{s \in \operatorname{Seq}(\kappa)}\left(B_{s} \backslash \bigcup_{\alpha<\kappa} B_{s-\alpha}\right) .
$$

Assume $x \notin \bigcup_{s}\left(B_{s} \backslash \bigcup_{\alpha<\kappa} B_{s \frown \alpha}\right)$. If we have for all $\alpha<\kappa$, that $x \notin B_{\langle\alpha\rangle}$, then $x \notin B$. And if there exists an $\alpha_{0}<\kappa$ such that $x \in B_{\left\langle\alpha_{0}\right\rangle}$, because $x \notin \bigcup_{s}\left(B_{s} \backslash \bigcup_{\alpha<\kappa} B_{s \frown \alpha}\right)$ we find an $\alpha_{1}$ such that $x \in B_{\left\langle\alpha_{0}, \alpha_{1}\right\rangle}$ and finally we find an $f \in \kappa^{\omega}$ such that for all $n \leq \omega: x \in B_{\bar{f}(n)}$. But this implies that $x \in A$. Now because $B_{s} \backslash \bigcup_{\alpha<\kappa} B_{s \frown \alpha} \subseteq B_{s} \backslash \bigcup_{\alpha<\kappa} A_{s \frown \alpha}=B_{s} \backslash A_{s}$ and because $\bigcup_{\alpha<\kappa} B_{s \frown \alpha}$ is the union of less than $\mathfrak{H}$ completely dual-Ramsey sets, $B_{s} \backslash \bigcup_{\alpha<\kappa} B_{s \frown \alpha}$ is completely dual-Ramsey and as a subset of $B_{s} \backslash A_{s}$, it is completely dual-Ramsey null. Therefore, $B \backslash A$ as a subset of the union of less than $\mathfrak{H}$ completely dual-Ramsey null sets is completely dual-Ramsey null and because $B$ is completely dual-Ramsey, $A$ is completely dual-Ramsey too.

REMARK: A similar result holds also for the Ramsey property and is proved by Matet in [Mt 2].

As a corollary we get a result which was first proved by Carlson and Simpson (cf. [CS]).

COROLLARY 3.6 Every analytic set is completely dual-Ramsey.

PRoOF: This follows from Theorem 3.5 and because each analytic set $A \subseteq$ $[\omega]^{\omega}$ can be written as

$$
A=\mathcal{A}\left\{Q_{s}: s \in \operatorname{Seq}(\omega)\right\}
$$

where each $Q_{s} \subseteq[\omega]^{\omega}$ is a closed set in the Baire space.

REMARK: For a similar result cf. [E]] or [Si].

\section{Game-families and the forcing notion $\mathbf{P}_{\mathfrak{F}}$}

First we define a game and game-families. Then we show that, for game-families $\mathfrak{F}$, the forcing notion $\mathbf{P}_{\mathfrak{F}}$ has pure decision and if $X$ is $\mathbf{P}_{\mathfrak{F}}$-generic and $Y \in(X)^{\omega}$, then $Y$ is $\mathbf{P}_{\mathfrak{F}^{-} \text {generic too. }}$ 
We call a family $\mathfrak{F} \subseteq(\omega)^{\omega}$ non-principal, if for all $X \in \mathfrak{F}$ there is a $Y \in \mathfrak{F}$ such that $Y \sqsubseteq X$ and $\neg(Y \stackrel{*}{=} X)$. A family $\mathfrak{F}$ is closed under refinement, if $X \sqsubseteq Y$ and $X \in \mathfrak{F}$ implies that $Y \in \mathfrak{F}$. Further it is closed under finite changes if for all $s \in(\mathbb{N})$ and $X \in \mathfrak{F}, X \sqcap s \in \mathfrak{F}$.

In the sequel $\mathfrak{F}$ is always a non-principal family which is closed under refinement and finite changes.

If $s \in(\mathbb{N})$ and $s \sqsubseteq X \in \mathfrak{F}$, then we call the dual Ellentuck neighborhood $(s, X)^{\omega}$ an $\mathfrak{F}$-dual Ellentuck neighborhood and write $(s, X)_{\mathfrak{F}}^{\omega}$ to emphasize that $X \in \mathfrak{F}$. A set $\mathcal{O} \subseteq(\omega)^{\omega}$ is called $\mathfrak{F}$-open if $\mathcal{O}$ can be written as the union of $\mathfrak{F}$-dual Ellentuck neighborhoods. For $s \in(\mathbb{N})$ remember that $s^{*}=s \cup\{\{\operatorname{dom}(s)\}\}$.

Fix a family $\mathfrak{F} \subseteq(\omega)^{\omega}$ (which is non-principal and closed under refinement and finite changes). Let $X \in \mathfrak{F}$ and $s \in(\mathbb{N})$ be such that $s \sqsubseteq X$. We associate with $(s, X)_{\mathfrak{F}}^{\omega}$ the following game. (This type of game was suggested first by Kastanas in $\mathrm{Ka}$.)

$$
\begin{array}{lcccc}
\text { I } & \left\langle X_{0}\right\rangle & \left\langle X_{1}\right\rangle & \multicolumn{2}{c}{\left\langle X_{2}\right\rangle} \\
& & & \\
\text { II } & \left\langle t_{0}, Y_{0}\right\rangle & \left\langle t_{1}, Y_{1}\right\rangle & \left\langle t_{2}, Y_{2}\right\rangle
\end{array}
$$

All the $X_{i}$ of player I and the $Y_{i}$ of player II must be elements of the family $\mathfrak{F}$. Player I plays $\left\langle X_{0}\right\rangle$ such that $X_{0} \in(s, X)_{\mathfrak{F}}^{\omega}$, then II plays $\left\langle t_{0}, Y_{0}\right\rangle$ such that $Y_{0} \in\left(s, X_{0}\right)_{\mathfrak{F}}^{\omega}, s \preceq t_{0}^{*} \preceq Y_{0}$ and $\left|t_{0}\right|=|s|$. For $n \geq 1$, the $n$th move of player $\mathrm{I}$ is $\left\langle X_{n}\right\rangle$ such that $X_{n} \in\left(t_{n-1}^{*}, Y_{n-1}\right)_{\mathfrak{F}}^{\omega}$ and then player II plays $\left\langle t_{n}, Y_{n}\right\rangle$ such that $Y_{n} \in\left(t_{n-1}^{*}, X_{n}\right)_{\mathfrak{F}}^{\omega}, t_{n-1}^{*} \preceq t_{n}^{*} \preceq Y_{n}$ and $\left|t_{n}\right|=\left|t_{n-1}\right|+1$. Player I wins iff the only $Y$ with $t_{n} \preceq Y$ (for all $n$ ) is in $\mathfrak{F}$. We denote this game by $\mathcal{G}(\mathfrak{F})$ starting with $\langle s, X\rangle$.

A non-principal family $\mathfrak{F}$ which is closed under refinement and finite changes is a game-family if player II has no winning strategy in the game $\mathcal{G}(\mathfrak{F})$.

A family $\mathfrak{F} \subseteq(\omega)^{\omega}$ is called a filter if for any $X, Y \in \mathfrak{F}$, also $X \sqcap Y \in \mathfrak{F}$. A filter which is also a game-family is called a game-filter. Note that $(\omega)^{\omega}$ is gamefamily but not a game-filter. (But it is consistent with ZFC that game-filters exist, as Theorem 5.1 will show).

Let $\mathcal{O} \subseteq(\omega)^{\omega}$ be an $\mathfrak{F}$-open set. Call $(s, X)_{\mathfrak{F}}^{\omega} \operatorname{good}$ (with respect to $\mathcal{O}$ ), if for some $Y \in(s, X)_{\mathfrak{F}}^{\omega} \cap \mathfrak{F},(s, Y)_{\mathfrak{F}}^{\omega} \subseteq \mathcal{O}$; otherwise call it bad. Note that if $(s, X)_{\mathfrak{F}}^{\omega}$ is bad and $Y \in(s, X)_{\mathfrak{F}}^{\omega} \cap \mathfrak{F}$, then $(s, Y)_{\mathfrak{F}}^{\omega}$ is bad, too. We call $(s, X)_{\mathfrak{F}}^{\omega}$ ugly if $\left(t^{*}, X\right)_{\mathfrak{F}}^{\omega}$ is bad for all $s \preceq t^{*} \sqsubseteq X$ with $|t|=|s|$. Note that if $(s, X)_{\mathfrak{F}}^{\omega}$ is ugly, then $(s, X)_{\mathfrak{F}}^{\omega}$ is bad, too.

To prove the following two lemmas, we will follow in fact the proof of Lemma 19.15 in $[\mathrm{Ke}$.

LEMMA 4.1 Let $\mathfrak{F}$ be a game-family and $\mathcal{O} \subseteq(\omega)^{\omega}$ an $\mathfrak{F}$-open set. If $(s, X)_{\mathfrak{F}}^{\omega}$ is bad (with respect to $\mathcal{O}$ ), then there exists a $Z \in(s, X)_{\mathfrak{F}}^{\omega}$ such that $(s, Z)_{\mathfrak{F}}^{\omega}$ is ugly.

PRoOF: We begin by describing a strategy for player II in the game $\mathcal{G}(\mathfrak{F})$ starting with $\langle s, X\rangle$. Let $\left\langle X_{n}\right\rangle$ be the $n$th move of player I and $t_{n}$ be such that $s \preceq t_{n},\left|t_{n}\right|=|s|+n$ and $t_{n}^{*} \preceq X_{n}$. Let $\left\{t_{n}^{i}: i \leq m\right\}$ be an enumeration of all $t$ 
such that $s \preceq t \sqsubseteq t_{n},|t|=|s|$ and $\operatorname{dom}(t)=\operatorname{dom}\left(t_{n}\right)$. Further let $Y^{-1}:=X_{n}$. Now choose for each $i \leq m$ a partition $Y^{i} \in \mathfrak{F}$ such that $Y^{i} \sqsubseteq Y^{i-1}, t_{n}^{*} \preceq Y^{i}$ and $\left(\left(t_{n}^{i}\right)^{*}, Y^{i}\right)_{\mathfrak{F}}^{\omega}$ is bad or $\left(\left(t_{n}^{i}\right)^{*}, Y^{i}\right)_{\mathfrak{F}}^{\omega} \subseteq \mathcal{O}$. Finally, let $Y_{n}:=Y^{m}$ and let player II play $\left\langle t_{n}, Y_{n}\right\rangle$.

Because player II has no winning strategy, player I can play so that the only $Y$ with $t_{n} \preceq Y$ (for all $n$ ) belongs to $\mathfrak{F}$. Let $S_{Y}:=\left\{t^{*} \sqsubseteq Y: s \preceq t \wedge|t|=\right.$ $|s|\}$; then (because of the strategy of player II), for all $t \in S_{Y}$ we have either $\left(t^{*}, Y\right)_{\mathfrak{F}}^{\omega}$ is bad or $\left(t^{*}, Y\right)_{\mathfrak{F}}^{\omega} \subseteq \mathcal{O}$. Now let $C_{0}:=\left\{t \in S_{Y}:(t, Y)_{\mathfrak{F}}^{\omega}\right.$ is bad $\}$ and $C_{1}:=\left\{t \in S_{Y}:\left(t^{*}, Y\right)_{\mathfrak{F}}^{\omega} \subseteq \mathcal{O}\right\}=S_{Y} \backslash C_{0}$. By a result of [HM, there exists a partition $Z \in(s, Y)_{\mathfrak{F}}^{\omega} \cap \mathfrak{F}$, such that $S_{Z} \subseteq C_{0}$ or $S_{Z} \subseteq C_{1}$. If we are in the latter case, we have $(s, Z)_{\mathfrak{\mathfrak { F }}}^{\omega} \subseteq \mathcal{O}$, which contradicts that $(s, X)_{\mathfrak{\mathfrak { F }}}^{\omega}$ is bad. So we must have $S_{Z} \subseteq C_{0}$, which implies that $(s, Z)_{\mathfrak{F}}^{\omega}$ is ugly and completes the proof of the Lemma.

LEMMA 4.2 If $\mathfrak{F}$ is a game-family and $\mathcal{O} \subseteq(\omega)^{\omega}$ is an $\mathfrak{F}$-open set, then for every $\mathfrak{F}$-dual Ellentuck neighborhood $(s, X)_{\mathfrak{F}}^{\omega}$ there exists a $Y \in(s, X)_{\mathfrak{F}}^{\omega} \cap \mathfrak{F}$ such that $(s, Y)_{\mathfrak{F}}^{\omega} \subseteq \mathcal{O}$ or $(s, Y)_{\mathfrak{F}}^{\omega} \cap \mathcal{O} \cap \mathfrak{F}=\emptyset$.

Proof: If $(s, X)_{\mathfrak{F}}^{\omega}$ is good, then we are done. Otherwise we consider the game $\mathcal{G}(\mathfrak{F})$ starting with $\langle s, X\rangle$. Let $\left\langle X_{0}\right\rangle$ be the first move of player I. Because $\left(s, X_{0}\right)_{\mathfrak{F}}^{\omega}$ is bad, by Lemma 4.1 we can choose $Y^{\prime} \in\left(s, X_{0}\right)_{\mathfrak{F}}^{\omega} \cap \mathfrak{F}$ such that $\left(s, Y^{\prime}\right)_{\mathfrak{\mathfrak { F }}}^{\boldsymbol{\omega}}$ is ugly. Let $t_{0}$ be such that $s \preceq t_{0}^{*} \preceq Y^{\prime}$ and $\left|t_{0}\right|=|s|$. Now we choose $Y_{0} \in\left(t_{0}^{*}, Y^{\prime}\right)_{\mathfrak{F}}^{\omega} \cap \mathfrak{F}$ such that $\left(t_{0}^{*}, Y_{0}\right)_{\mathfrak{F}}^{\omega}$ is ugly, which is is possible because $\left(t_{0}, Y^{\prime}\right)_{\mathfrak{F}}^{\omega}$ is ugly and therefore $\left(t_{0}^{*}, Y^{\prime}\right)_{\mathfrak{F}}^{\omega}$ is bad. Note that for all $t$ with $s \preceq t \sqsubseteq t_{0}$ and $\operatorname{dom}(t)=\operatorname{dom}\left(t_{0}\right)$ we have $\left(t^{*}, Y_{0}\right)_{\mathfrak{F}}^{\omega}$ is ugly. Now player II plays $\left\langle t_{0}, Y_{0}\right\rangle$.

Let $\left\langle X_{n+1}\right\rangle$ be the $(n+1)$ th move of player I. By the strategy of player II we have $\left(t^{*}, X_{n+1}\right)_{\mathfrak{F}}^{\omega}$ is ugly for all $t$ with $s \preceq t \sqsubseteq t_{n}$ and $\operatorname{dom}(t)=\operatorname{dom}\left(t_{n}\right)$. Let $t_{n+1}$ be such that $\left|t_{n+1}\right|=\left|t_{n}\right|+1=|s|+n$ and $t_{n}^{*} \preceq t_{n+1}^{*} \preceq X_{n+1}$. Let $\left\{t_{n+1}^{i}: i \leq m\right\}$ be an enumeration of all $t$ such that $s \preceq t \sqsubseteq t_{n+1}$ and $\operatorname{dom}(t)=\operatorname{dom}\left(t_{n+1}\right)$. Further let $Y^{-1}:=X_{n+1}$. Now choose for each $i \leq m$ a partition $Y^{i} \in \mathfrak{F}$ such that $Y^{i} \sqsubseteq Y^{i-1}, t_{n+1}^{*} \preceq Y^{i}$ and $\left(\left(t_{n+1}^{i}\right)^{*}, Y^{i}\right)_{\mathfrak{F}}^{\omega}$ is ugly. (This is possible because we know that $\left(t^{*}, X_{k}\right)_{\mathfrak{F}}^{\omega}$ is ugly for all $k \leq n$ and $t$ with $s \preceq t \sqsubseteq t_{k}$ and $\operatorname{dom}(t)=\operatorname{dom}\left(t_{k}\right)$, which implies that $\left(\left(t_{n+1}^{i}\right)^{*}, X_{n+1}\right)_{\mathfrak{F}}^{\omega}$ is bad.) Finally, let $Y_{n+1}:=Y^{m}$ and let player II play $\left\langle t_{n+1}, Y_{n+1}\right\rangle$.

Because player II has no winning strategy, player I can play so that the only $Y$ with $t_{n} \preceq Y$ (for all $n$ ) belongs to $\mathfrak{F}$. We claim that $(s, Y)_{\mathfrak{F}}^{\omega} \cap \mathcal{O} \cap \mathfrak{F}=\emptyset$. Let $Z \in(s, Y)_{\mathfrak{F}}^{\omega} \cap \mathcal{O} \cap \mathfrak{F}$. Because $\mathcal{O}$ is $\mathfrak{F}$-open we find a $t \preceq Z$ such that $\left(t^{*}, Z\right)_{\mathfrak{\mathfrak { F }}}^{\omega} \subseteq \mathcal{O}$. Because $t^{*} \sqsubseteq Y$ we know by the strategy of player II that $\left(t^{*}, Y\right)_{\mathfrak{F}}^{\omega}$ is bad. Hence, there is no $Z \in\left(t^{*}, Y\right)_{\mathfrak{F}}^{\omega}$ such that $\left(t^{*}, Z\right)_{\mathfrak{F}}^{\omega} \subseteq \mathcal{O}$. This completes the proof.

Now we give two properties of the forcing notion $\mathbf{P}_{\mathfrak{F}}$, (where $\mathbf{P}_{\mathfrak{F}}$ is defined as in section 2 and $\mathfrak{F}$ is a game-family). Note that for $\mathfrak{F}=(\omega)^{\omega}$ (which is obviously a game-family) the forcing notion $\mathbf{P}_{\mathfrak{F}}$ is the same as dual-Mathias forcing. The first property of the forcing notion $\mathbf{P}_{\mathfrak{F}}$ we give is called pure decision.

THEOREM 4.3 Let $\mathfrak{F}$ be a game-family and let $\Phi$ be a sentence of the forcing language $\mathbf{P}_{\mathfrak{F}}$. For any $\mathbf{P}_{\mathfrak{F}}$-condition $(s, X)_{\mathfrak{F}}^{\omega}$ there exists a $\mathbf{P}_{\mathfrak{F}}$-condition $(s, Y)_{\mathfrak{F}}^{\omega} \leq(s, X)_{\mathfrak{F}}^{\omega}$ such that $(s, Y)_{\mathfrak{F}}^{\omega} \Vdash \mathbf{P}_{\mathfrak{F}} \Phi$ or $(s, Y)_{\mathfrak{F}}^{\omega} \vdash_{\mathbf{P}_{\mathfrak{F}}} \neg \Phi$. 
Proof: With respect to $\Phi$ we define $\mathcal{O}_{1}:=\left\{Y:(t, Y)_{\mathfrak{F}}^{\omega} \Vdash_{\mathbf{P}_{\mathfrak{F}}} \Phi\right.$ for some $\left.t \preceq Y \in \mathfrak{F}\right\}$ and $\mathcal{O}_{2}:=\left\{Y:(t, Y)_{\widetilde{F}}^{\omega} \Vdash \mathbf{P}_{\mathfrak{F}} \neg \Phi\right.$ for some $\left.t \preceq Y \in \mathfrak{F}\right\}$. Clearly $\mathcal{O}_{1}$ and $\mathcal{O}_{2}$ are both $\mathfrak{F}$-open and $\mathcal{O}_{1} \cup \mathcal{O}_{2}$ is even dense (with respect to the partial order $\mathbf{P}_{\mathfrak{F}}$ ). Because $\mathfrak{F}$ is a game-family, by Lemma 4.2 we know that for any $(s, X)_{\mathfrak{F}}^{\omega} \in \mathbf{P}_{\mathfrak{D}}$ there exists $Y \in(s, X)_{\mathfrak{F}}^{\omega} \cap \mathfrak{F}$ such that either $(s, Y)_{\mathfrak{F}}^{\omega} \subseteq \mathcal{O}_{1}$ or $(s, Y)_{\mathfrak{F}}^{\omega} \cap \mathcal{O}_{1} \cap \mathfrak{F}=\emptyset$. In the former case we have $(s, Y)_{\mathfrak{F}}^{\omega} \Vdash \vdash_{\mathbf{P}_{\mathfrak{F}}} \Phi$ and we are done. In the latter case we find $Y^{\prime} \in(s, Y)_{\mathfrak{F}}^{\omega} \cap \mathfrak{F}$ such that $\left(s, Y^{\prime}\right)_{\mathfrak{F}}^{\omega} \subseteq \mathcal{O}_{2}$. (Otherwise we would have $\left(s, Y^{\prime}\right)_{\mathfrak{F}}^{\omega} \cap\left(\mathcal{O}_{2} \cup \mathcal{O}_{1}\right) \cap \mathfrak{F}=\emptyset$, which is impossible by the density of $\mathcal{O}_{1} \cup \mathcal{O}_{2}$.) Hence, $\left(s, Y^{\prime}\right)_{\mathfrak{F}}^{\omega} \Vdash \mathbf{P}_{\mathfrak{F}} \neg \Phi$.

Let $\mathfrak{F}$ be a game-family, $\mathfrak{G}$ be $\mathbf{P}_{\mathfrak{F}}$-generic and define $X_{\mathfrak{G}}:=\bigcap \mathfrak{G}$. Now $X_{\mathfrak{G}}$ is an infinite partition and $\mathfrak{G}=\left\{(s, Z)_{\mathfrak{F}}^{\omega}: s \preceq X_{\mathfrak{G}} \sqsubseteq Z\right\}$. Therefore we can consider the partition $X_{\mathfrak{G}} \in(\omega)^{\omega}$ as a $\mathbf{P}_{\mathfrak{F}}$-generic object. Further we have $\mathfrak{G} \subseteq \mathbf{P}_{\mathfrak{F}}$ is $\mathbf{P}_{\mathfrak{F}}$-generic if and only if $X_{\mathfrak{G}} \in \bigcup D$ for all $D \subseteq \mathbf{P}_{\mathfrak{F}}$ which are dense in $\mathbf{P}_{\mathfrak{F}}$. Note that if $D$ is dense in $\mathbf{P}_{\mathfrak{F}}$, then $\bigcup D$ is $\mathfrak{F}$-open.

The next theorem shows in fact that if $\mathfrak{F}$ is a game-family, then $\mathbf{P}_{\mathfrak{F}}$ is proper.

THEOREM 4.4 Let $\mathfrak{F} \subseteq(\omega)^{\omega}$ be a game-family. If $X_{0} \in(\omega)^{\omega}$ is $\mathbf{P}_{\mathfrak{F}^{-}}$generic over $V$ and $Y_{0} \in\left(X_{0}\right)^{\omega} \cap V\left[X_{0}\right]$, then $Y_{0}$ is also $\mathbf{P}_{\mathfrak{F}^{-}}$generic over $V$.

Proof: Take an arbitrary dense set $D \subseteq \mathbf{P}_{\mathfrak{F}}$, i.e. for all $(s, X)_{\mathfrak{F}}^{\omega}$ there exists a $(t, Y)_{\mathfrak{F}}^{\omega} \subseteq(s, X)_{\mathfrak{F}}^{\omega}$ such that $(t, Y)_{\mathfrak{F}}^{\omega} \in D$. Let $D^{\prime}$ be the set of all $(s, Z)_{\mathfrak{F}}^{\omega}$ such that $(t, Z)_{\mathfrak{F}}^{\omega} \subseteq \bigcup D$ for all $t \sqsubseteq s$ with $\operatorname{dom}(t)=\operatorname{dom}(s)$.

First we show that $D^{\prime}$ is dense in $\mathbf{P}_{\mathfrak{F}}$. For this take an arbitrary $(s, W)_{\mathfrak{F}}^{\omega}$ and let $\left\{t_{i}: 0 \leq i \leq m\right\}$ be an enumeration of all $t \in(\mathbb{N})$ such that $t \sqsubseteq s$ and $\operatorname{dom}(t)=\operatorname{dom}(s)$. Because $D$ is dense in $\mathbf{P}_{\mathfrak{F}}$ and $\bigcup D$ is $\mathfrak{F}$-open, we find for every $t_{i}$ a $W^{\prime} \in \mathfrak{F}$ such that $t_{i} \sqsubseteq W^{\prime}$ and $\left(t_{i}, W^{\prime}\right)_{\mathfrak{F}}^{\omega} \subseteq \bigcup D$. Moreover, if we define $W_{-1}:=W$, we can choose for every $i \leq m$ a partition $W_{i} \in \mathfrak{F}$ such that $W_{i} \sqsubseteq W_{i-1}, s \preceq W_{i}$ and $\left(t_{i}, W_{i}\right)_{\mathfrak{F}}^{\omega} \subseteq \bigcup D$. Now $\left(s, W_{m}\right)_{\mathfrak{F}}^{\omega} \in D^{\prime}$ and because $\left(s, W_{m}\right)_{\mathfrak{F}}^{\omega} \subseteq(s, W)_{\mathfrak{F}}^{\omega}, D^{\prime}$ is dense in $\mathbf{P}_{\mathfrak{F}}$.

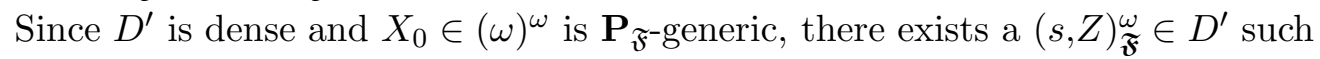
that $s \preceq X_{0} \sqsubseteq Z$. Because $Y_{0} \in\left(X_{0}\right)^{\omega}$ we have $t \preceq Y_{0} \sqsubseteq Z$ for some $t \sqsubseteq s$ and because $(t, Z)_{\mathfrak{F}}^{\omega} \subseteq \bigcup D$, we get $Y_{0} \in \bigcup D$. Hence, $Y_{0} \in \bigcup D$ for every dense $D \subseteq \mathbf{P}_{\mathfrak{F}}$, which completes the proof.

REMARK: A similar result is proved in Ma and Mt 3.

\section{On the dual-Mathias forcing and game-filters}

Now we show that it is consistent with ZFC that game-filters exist. (Remember that a game-filter $\mathfrak{F}$ is a game-family which is also a filter and a game-family is a non-principal family which is closed under refinement and finite changes such that player II has no winning strategy in the game $\mathcal{G}(\mathfrak{F})$.) Further we show that the dual-Mathias forcing $\mathfrak{M}$ is flexible and with this result we can prove that if $V$ is $\Sigma_{4}^{1}-\mathfrak{M}$-absolute, then $\omega_{1}^{V}$ is inaccessible in L.

In the sequel let $\mathfrak{U}$ be the forcing notion we defined in section 2 .

THEOREM 5.1 If $\mathfrak{D}$ is $\mathfrak{U}$-generic over $V$, then $\mathfrak{D}$ is a game-filter in $V[\mathfrak{D}]$ with respect to the game $\mathcal{G}(\mathfrak{D})$. 
Proof: Because $\mathfrak{D}$ is $\mathfrak{U}$-generic over $V$, we know that $\mathfrak{D} \subseteq(\omega)^{\omega}$ is a nonprincipal family in $V[\mathfrak{D}]$ which is closed under refinement and finite changes, and for $X, Y \in \mathfrak{D}$ we also have $X \sqcap Y \in \mathfrak{D}$. It remains to show that player II has no winning strategy in the game $\mathcal{G}(\mathfrak{D})$.

Let $\tilde{\sigma}$ be a $\mathfrak{U}$-name for a strategy for player II in the game $\mathcal{G}(\tilde{\mathfrak{D}})$, where $\tilde{\mathfrak{D}}$ is the canonical $\mathfrak{U}$-name for the $\mathfrak{U}$-generic object. Let us assume that player II will follow this strategy. We may assume that

$$
\mathbb{1} \Vdash_{\mathfrak{U}} \text { “ } \tilde{\sigma} \text { is a strategy for II in the game } \mathcal{G}(\tilde{\mathfrak{D}}) \text { ". }
$$

If

$$
Z \Vdash \mathfrak{U} \tilde{\sigma}\left(\left\langle\tilde{X}_{0}\right\rangle,\left\langle\tilde{t}_{0}, \tilde{Y}_{0}\right\rangle, \ldots,\left\langle\tilde{X}_{n}\right\rangle\right)=\left\langle\tilde{t}_{n}, \tilde{Y}_{n}\right\rangle,
$$

then for $n \geq 1$ we get

$$
Z \Vdash \mathfrak{U}\left(\left|\tilde{t}_{n}\right|=\left|\tilde{t}_{n-1}\right|+1 \wedge \tilde{t}_{n-1}^{*} \preceq \tilde{t}_{n}^{*} \preceq \tilde{Y}_{n} \sqsubseteq \tilde{X}_{n} \wedge \tilde{Y}_{n} \in \tilde{\mathfrak{D}}\right)
$$

and for $n=0$ we have

$$
Z \Vdash \mathfrak{U}\left(\left|\tilde{t}_{0}\right|=|\tilde{s}| \wedge \tilde{s} \preceq \tilde{t}_{0}^{*} \preceq \tilde{Y}_{0} \sqsubseteq \tilde{X}_{0} \sqsubseteq \tilde{X} \wedge \tilde{Y}_{0} \in \tilde{\mathfrak{D}}\right)
$$

where $\langle\tilde{s}, \tilde{X}\rangle$ is the starting point of $\mathcal{G}(\tilde{\mathfrak{D}})$.

Now let $\langle\tilde{s}, \tilde{X}\rangle$ (the starting point of the game $\mathcal{G}(\tilde{\mathfrak{D}})$ ) be such that $(\tilde{s}, \tilde{X})^{\omega}$ is a $\mathfrak{U}$-name for a dual Ellentuck neighborhood and let $Z_{0} \in(\omega)^{\omega} \cap V$ be a $\mathfrak{U}$ condition in $V$ such that $Z_{0} \Vdash \mathfrak{U} \tilde{X} \in \tilde{\mathfrak{D}}$. Therefore, $Z_{0} \Vdash \mathfrak{U}$ " $(\tilde{s}, \tilde{X})^{\omega}$ is a $\tilde{\mathfrak{D}}$-dual Ellentuck neighborhood". By Fact 2.3 we know that the forcing notion $\mathfrak{U}$ adds no new reals (and therefore no new partitions) to $V$. So, we find a $Z_{0}^{\prime} \sqsubseteq{ }^{*} Z_{0}$ and a dual Ellentuck neighborhood $(s, X)^{\omega}$ in $V$ such that

$$
Z_{0}^{\prime} \Vdash \mathfrak{U}\langle\tilde{s}, \tilde{X}\rangle=\langle\check{s}, \check{X}\rangle
$$

where $\check{s}$ and $\check{X}$ are the canonical $\mathfrak{U}$-names for $s$ and $X$. Because $Z_{0}^{\prime} \Vdash \mathfrak{U} \check{X} \in \tilde{\mathfrak{D}}$, we must have $Z_{0}^{\prime} \leq X$, which is the same as $Z_{0}^{\prime} \sqsubseteq^{*} X$. Finally put $X_{0} \in(\omega)^{\omega}$ such that $X_{0} \stackrel{*}{=} Z_{0}^{\prime}$ and $X_{0} \in(s, X)^{\omega}$. Player I plays now $\left\langle\check{X}_{0}\right\rangle$. Since player II follows the strategy $\tilde{\sigma}$, player II plays now $\tilde{\sigma}\left(\left\langle\tilde{X}_{0}\right\rangle\right)=:\left\langle\tilde{t}_{0}, \tilde{Y}_{0}\right\rangle$. Again by Fact 2.3 there exists a $Z_{1} \sqsubseteq^{*} X_{0}$ and a dual Ellentuck neighborhood $\left(t_{0}, Y_{0}\right)^{\omega}$ in $V$ such that

$$
Z_{1} \Vdash \mathfrak{U}\left\langle\tilde{t}_{0}, \tilde{Y}_{0}\right\rangle=\left\langle\check{t}_{0}, \check{Y}_{0}\right\rangle
$$

And again by $Z_{1} \Vdash \mathfrak{U} \check{Y}_{0} \in \tilde{\mathfrak{D}}$ we find $X_{1} \stackrel{*}{=} Z_{1}$ such that $t_{0}^{*} \preceq X_{1} \sqsubseteq Y_{0}$. Player I plays now $\left\langle\check{X}_{1}\right\rangle$.

In general, if $\tilde{\sigma}\left(\left\langle\tilde{X}_{0}\right\rangle,\left\langle\tilde{t}_{0}, \tilde{Y}_{0}\right\rangle, \ldots,\left\langle\tilde{X}_{n}\right\rangle\right)=\left\langle\tilde{t}_{n}, \tilde{Y}_{n}\right\rangle$, then player I can play $\check{X}_{n+1}$ such that $X_{n} \Vdash \mathfrak{U}\left\langle\tilde{t}_{n}, \tilde{Y}_{n}\right\rangle=\left\langle\check{t}_{n}, \check{Y}_{n}\right\rangle$ and $t_{n}^{*} \preceq X_{n+1} \sqsubseteq Y_{n}$. For $n \geq m$ we also have $X_{n} \sqsubseteq X_{m}$. Let $Y \in(\omega)^{\omega}$ be the such that $t_{n} \preceq Y$ (for all $n$ ), then

$$
Y \Vdash \mathfrak{U} \text { "the only } \tilde{Y} \text { such that } \tilde{t}_{n} \preceq \tilde{Y} \text { (for all } n \text { ) is in } \tilde{\mathfrak{D}} \text { ". }
$$

Hence, the strategy $\tilde{\sigma}$ is not a winning strategy for player II and because $\tilde{\sigma}$ was an arbitrary strategy, player II has no winning strategy at all.

REMARK: A similar result is in fact proved in [Ma] (cf. also [Mt 2]).

As a corollary we get that the forcing notion $\mathbf{P}_{\mathfrak{D}}$ (where $\mathfrak{D}$ is $\mathfrak{U}$-generic over $V)$ has pure decision in $V[\mathfrak{D}]$. 
COROLlARY 5.2 Let $\mathfrak{D}$ be $\mathfrak{U}$-generic over $V$. Then the forcing notion $\mathbf{P}_{\mathfrak{D}}$ has pure decision in $V[\mathfrak{D}]$.

Proof: This follows from Theorem 4.3 and Theorem 5.1.

Corollary 5.2 follows also from the facts that the dual-Mathias forcing has pure decision (cf. CSA ) and that it can be written as a two step iteration as in section 2 .

REMARK: If $D$ is U-generic over $V$, then $\mathbf{P}_{D}$ has pure decision in $V[D]$ (cf. Ma]).

\section{Some more properties of $\mathfrak{M}$}

Let $\mathbf{P}$ be a notion of forcing in the model $V$. We say that $V$ is $\Sigma_{n}^{1}-\mathbf{P}$-absolute if for every $\Sigma_{n}^{1}$-sentences $\Phi$ with parameters in $V$ the following is true.

$$
V \models \Phi \text { if and only if } V[G] \models \Phi,
$$

where $G$ is any $\mathbf{P}$-generic object over $V$.

Now we will show that if $V$ is $\Sigma_{4}^{1}$ - $\mathfrak{M}$-absolute, then $\omega_{1}^{V}$ is inaccessible in $L$. For this we first will translate the dual-Mathias forcing in a tree forcing notion.

If $s$ is a partial partition of some natural number $n \in \omega$, then we can consider $s$ as a subset of $\mathcal{P}(n)$ or equivalently, as a finite set of finite sets of natural numbers. Let $t$ be a finite set of natural numbers, then $\sharp t$ is such that for all $k \in \omega: \operatorname{div}\left(\sharp t, 2^{k}\right)$ is odd $\Leftrightarrow k \in s$. (Remember that $\operatorname{div}(n, m):=\max (\{k \in \omega:$ $k \cdot m \leq n\}$.) Now $\sharp s$ is such that for all $k \in \omega: \operatorname{div}\left(\sharp s, 2^{k}\right)$ is odd $\Leftrightarrow k=\sharp t$ for some $t \in s$. (In fact $\sharp s$ is defined for any finite set of finite sets of natural numbers.) If $s \in(\mathbb{N})$, then $|s|$ denotes the cardinality of $s$, which is the number of blocks of $s$.

For $s \in(\mathbb{N})$ with $|s|=k$ let $\bar{s}$ be the finite sequence $\left\langle n_{1}, \ldots, n_{k}\right\rangle$ where $n_{i}:=\sharp s_{i}$ and $s_{i} \in(\mathbb{N})$ is such that $\left|s_{i}\right|=i$ and $s_{i}^{*} \preceq s^{*}$.

Now let $p=(s, X)^{\omega}$ be an $\mathfrak{M}$-condition. Without loss of generality we may assume that $s^{*} \sqsubseteq X$. The tree $\mathfrak{t}_{p} \subseteq \omega^{<\omega}$ is defined as follows.

$$
\sigma \in \mathfrak{t}_{p} \Leftrightarrow \exists t \in(\mathbb{N})\left(\left(t^{*} \preceq s^{*} \vee s \preceq t\right) \wedge t^{*} \sqsubseteq X \wedge \sigma=\bar{t} .\right.
$$

FACT 5.3 Let $p, q$ be two $\mathfrak{M}$-conditions. Then $\mathfrak{t}_{p}$ is a subtree of $\mathfrak{t}_{q}$ if and only if $p \leq q \cdot \dashv$

Finally let $T_{\mathfrak{M}}:=\left\{\mathfrak{t}_{p}: p \in \mathfrak{M}\right\}$; then $T_{\mathfrak{M}}$ is a set of trees. We stipulate that $\mathfrak{t}_{p} \leq \mathfrak{t}_{q}$ if $\mathfrak{t}_{p}$ is a subtree of $\mathfrak{t}_{q}$. Then (by Fact 5.3) forcing with $\mathfrak{T}_{\mathfrak{M}}:=\left\langle T_{\mathfrak{M}}, \leq\right\rangle$ is the same as forcing with $\mathfrak{M}$.

Now we will give the definition of a flexible forcing notion $\mathbf{P}$. But first we have to give some other definitions.

A set $T \subseteq \omega^{<\omega}$ is called a Laver-tree if

$T$ is a tree and $\exists \tau \in T \forall \sigma \in T(\sigma \subseteq \tau \vee(\tau \subseteq \sigma \wedge|\{n: \sigma \frown n \in T\}|=\omega))$. 
(We call $\tau$ the stem of $T$. For $\sigma \in T$ we let $\operatorname{succ}_{T}(\sigma):=\{n: \sigma \frown n \in T\}$, (the successors of $\sigma$ in $T$ ) and $T_{\rho}:=\{\sigma \in T: \sigma \subseteq \rho \wedge \rho \subseteq \sigma\}$.)

For a Laver-tree $T$, we say $A \subseteq T$ is a front if $\sigma \neq \tau$ in $A$ implies $\sigma \nsubseteq \tau$ and for all $f \in[T]$ there is an $n \in \omega$ such that $\left.f\right|_{n} \in A$.

The meaning of $p \leq \llbracket \Phi \rrbracket$ and $p \cap \llbracket \Phi \rrbracket$ are $U_{p} \subseteq \llbracket \Phi \rrbracket$ and $U_{p} \cap \llbracket \Phi \rrbracket$, respectively.

1. We say a forcing notion $\mathbf{P}$ is Laver-like if there is a $\mathbf{P}$-name $\tilde{r}$ for a dominating real such that

(i) the complete Boolean algebra generated by the family $\{\llbracket \tilde{r}(i)=n \rrbracket$ : $i, n \in \omega\}$ equals r.o. $(\mathbf{P})$, and

(ii) for each condition $p \in \mathbf{P}$ there exists a Laver-tree $T \subseteq \omega^{<\omega}$ so that

$\forall \sigma \in T\left(p\left(T_{\sigma}\right):=\prod_{n \in \omega} \sum_{\tau \in T_{\sigma}}\left\{\left.p \cap \llbracket \tilde{r}\right|_{\lg (\tau)}=\tau \rrbracket: \lg (\tau)=n\right\} \in\right.$ r.o. $\left.(\mathbf{P}) \backslash\{\mathbf{0}\}\right)$.

We express this by saying $p(T) \neq \emptyset$ where $p(T):=p\left(T_{\text {stem }(T)}\right)$.

2. If $\tilde{r}$ is a $\mathbf{P}$-name that witnesses that $\mathbf{P}$ is Laver-like, we say that $\mathbf{P}$ has strong fusion if for countably many open dense sets $D_{n} \subseteq \mathbf{P}$ and for $p \in \mathbf{P}$, there is a Laver-tree $T$ such that $p(T) \neq \emptyset$ and for each $n$ :

$$
\left\{\sigma \in T:\left.p(T) \cap \llbracket \tilde{r}\right|_{\lg (\sigma)}=\sigma \rrbracket \in D_{n}\right\}
$$

contains a front.

3. A Laver-like $\mathbf{P}$ is closed under finite changes if given a $p \in \mathbf{P}$ and Laver trees $T$ and $T^{\prime}$ so that for all $\sigma \in T^{\prime}:\left|\operatorname{succ}_{T}(\sigma) \backslash \operatorname{succ}_{T^{\prime}}(\sigma)\right|<\omega$, if $p(T) \neq \emptyset$, then $p\left(T^{\prime}\right) \neq \emptyset$, too.

Now we call $\mathbf{P}$ a flexible forcing notion iff $\mathbf{P}$ is Laver-like, has strong fusion and is closed under finite changes.

With this definition we can show (as a further symmetry between the forcing notions $\mathbf{M}$ and $\mathfrak{M})$, that the dual-Mathias forcing $\mathfrak{M}$ is flexible.

LEMMA 5.4 The dual-Mathias forcing $\mathfrak{M}$ is flexible.

PROOF: By $\mathfrak{M} \approx \mathfrak{T}_{\mathfrak{M}}$ it is enough to prove that the forcing notion $\mathfrak{T}_{\mathfrak{M}}$ is flexible. Let $\tilde{r}$ be the canonical $\mathfrak{T}_{\mathfrak{M}}$-name for the $\boldsymbol{T}_{\mathfrak{M}}$-generic object. By the definition of $\sharp$ and the construction of $\boldsymbol{T}_{\mathfrak{M}}, \tilde{r}$ is a name for a dominating real. The rest of the proof is similar to the proof that Mathias forcing is flexible, which is given in [HJ].

If all $\Sigma_{n}^{1}$-sets in $V$ with parameters in $V \cap W$ have the Ramsey property $\mathcal{R}$ or the dual-Ramsey property $\mathfrak{R}$, we will write $V \models \Sigma_{n}^{1}(\mathcal{R})_{W}$ or $V \models \Sigma_{n}^{1}(\mathfrak{R})_{W}$, respectively. If $V=W$, then we omit the index $W$. The notations for $\Delta_{n}^{1}$-sets and $\Pi_{n}^{1}$-sets are similar. Further $\mathcal{B}$ stands for the Baire property and $\mathcal{L}$ stands for Lebesgue measurable.

Now we can prove the following

THEOREM 5.5 If $V$ is $\Sigma_{4}^{1}$ - $\mathfrak{M}$-absolute, then $\omega_{1}^{V}$ is inaccessible in $L$. 
PRoOF: To prove the corresponding result for Mathias forcing (cf. [HJ]) we used only that $\mathbf{M}$ is flexible and that, if $V$ is $\Sigma_{4}^{1}$-M-absolute, then $V \models \Sigma_{2}^{1}(\mathcal{R})$, which is the same as $\Sigma_{3}^{1}$-M-absoluteness (cf. [H]]). Therefore it is enough to prove that $\Sigma_{3}^{1}-\mathfrak{M}$-absoluteness implies $\Sigma_{3}^{1}$-M-absoluteness. It follows immediately from Fact 2.6 that $V \subseteq V^{\mathbf{M}} \subseteq V^{\mathfrak{M}}$. Now because $\Sigma_{3}^{1}$-formulas are upwards absolute, this completes the proof.

\section{Iteration of dual-Mathias forcing}

In this section we will build two models in which every $\Sigma_{2}^{1}$-set is dual-Ramsey. In the first model $2^{\aleph_{0}}=\aleph_{1}$ and in the second model $2^{\aleph_{0}}=\aleph_{2}$. With the result that dual-Mathias forcing has the Laver property we can show that $\Sigma_{2}^{1}(\mathfrak{R})$ implies neither $\Sigma_{2}^{1}(\mathcal{L})$ nor $\Sigma_{2}^{1}(\mathcal{B})$.

In the sequel we will use the same notations as in section 5 .

First we give a result similar to Theorem 1.15 of [JS].

LEMMA 6.1 Let $\mathfrak{D}$ be $\mathfrak{U}$-generic over $V$. If $\mathfrak{m}$ is $\mathbf{P}_{\mathfrak{D}}$-generic over $V[\mathfrak{D}]$, then $V[\mathfrak{D}][\mathfrak{m}] \models \Sigma_{2}^{1}(\mathfrak{R})_{\mathfrak{V}}$.

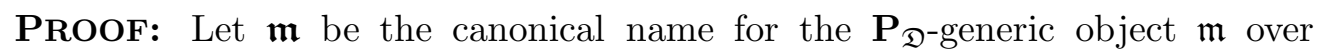
$V[\mathfrak{D}]$ and let $\varphi(X)$ be a $\Sigma_{2}^{1}$-formula with parameters in $V$. By Theorem 5.1 and Corollary 5.2, the forcing notion $\mathbf{P}_{\mathfrak{D}}$ has pure decision. So, there ex-

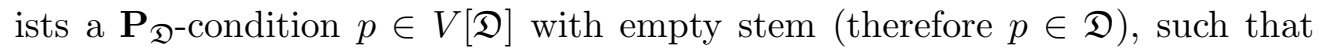
$V[\mathfrak{D}] \models " p \vdash_{\mathbf{P}_{\mathfrak{D}}} \varphi(\mathfrak{m})$ " or $V[\mathfrak{D}] \models " p \vdash_{\mathbf{P}_{\mathfrak{D}}} \neg \varphi(\mathfrak{m}) "$. Assume the former case holds. Because $\mathfrak{m} \sqsubseteq^{*} \mathfrak{q}$ for all $q \in \mathfrak{D}$, there is an $f \in[\omega]^{<\omega}$ such that $\mathfrak{m} \sqcap\{f\} \sqsubseteq p$. By Theorem 5.1 and Theorem 4.4 we know that if $X$ is $\mathbf{P}_{\mathfrak{D}^{-}}$ generic over $V[\mathfrak{D}]$ and $X^{\prime} \in(X)^{\omega} \cap V[\mathfrak{D}][\mathfrak{m}]$, then $X^{\prime}$ is also $\mathbf{P}_{\mathfrak{D}}$-generic over $V[\mathfrak{D}]$. Hence every $\mathfrak{m}^{\prime} \sqsubseteq \mathfrak{m} \sqcap\{\mathfrak{f}\} \sqsubseteq \mathfrak{p}$ is $\mathbf{P}_{\mathfrak{D}}$-generic over $V[\mathfrak{D}]$ and therefore $V[\mathfrak{D}]\left[\mathfrak{m}^{\prime}\right] \models \varphi\left(\mathfrak{m}^{\prime}\right)$. Because $\Sigma_{2}^{1}$-formulas are absolute, we get $V[\mathfrak{D}][\mathfrak{m}] \models \varphi\left(\mathfrak{m}^{\prime}\right)$. So, $V[\mathfrak{D}][\mathfrak{m}] \models \exists X \forall Y \in(X)^{\omega} \varphi(Y)$. The case when $V[\mathfrak{D}] \models$ " $p \Vdash_{\mathbf{P}_{\mathfrak{D}}} \neg \varphi(\mathfrak{m})$ " is similar. Hence, we finally have $V[\mathfrak{D}][\mathfrak{m}] \models \Sigma_{2}^{1}(\mathfrak{R})_{V}$.

REMARK: The proof of the analogous result can be found in [JS.

Because Gödel's constructible universe $L$ has a $\Delta_{2}^{1}$-well-ordering of the reals, $L$ is neither a model for $\Delta_{2}^{1}(\mathfrak{R})$ nor a model for $\Delta_{2}^{1}(\mathcal{R})$. But we can build a model in which $2^{\aleph_{0}}=\aleph_{1}$ and all $\Sigma_{2}^{1}$-sets are dual-Ramsey.

THEOREM 6.2 If we make an $\omega_{1}$-iteration of dual-Mathias forcing with countable support starting from $L$, we get a model in which every $\Sigma_{2}^{1}$-set of reals is dual-Ramsey and $2^{\aleph_{0}}=\aleph_{1}$.

Proof: Follows immediately from the Fact 2.5, Lemma 6.1 and the fact that the dual-Mathias forcing is proper.

REMARK: The proof of a similar result can be found in Ju.

We can build also a model in which $2^{\aleph_{0}}=\aleph_{2}$ and all $\Sigma_{2}^{1}$-sets are dual-Ramsey.

THEOREM 6.3 If we make an $\omega_{2}$-iteration of dual-Mathias forcing with countable support starting from $L$, we get a model in which every $\Sigma_{2}^{1}$-set of reals is dual-Ramsey and $2^{\aleph_{0}}=\aleph_{2}$. 
Proof: In Ha it is shown that a $\omega_{2}$-iteration of dual-Mathias forcing with countable support starting from $L$ yields a model in which $2^{\aleph_{0}}=\aleph_{2}$ and the union of fewer than $\aleph_{2}$ completely dual-Ramsey sets is completely dual-Ramsey. Now because each $\Sigma_{2}^{1}$-set can be written as the union of $\aleph_{1}$ analytic sets (and analytic sets are completely dual-Ramsey) all the $\Sigma_{2}^{1}$-sets are dual-Ramsey.

REMARK: A similar result is true because an $\omega_{2}$-iteration of Mathias forcing with countable support starting from $L$ yields a model in which $\mathfrak{h}=\aleph_{2}$ (cf. SSS), and $\mathfrak{h}$ can be considered as the additivity of the ideal of completely Ramsey null sets (cf. [P] $]$ ).

For the next result we have to give first the definition of the Laver property.

A cone $\bar{A}$ is a sequence $\left\langle A_{k}: k \in \omega\right\rangle$ of finite subsets of $\omega$ with $\left|A_{k}\right|<2^{k}$. We say that $\bar{A}$ covers a function $f \in \omega^{\omega}$ if for all $k>0: f(k) \in A_{k}$. For a function $H \in \omega^{\omega}$, we write $\Pi H$ for the set $\left\{f \in \omega^{\omega}: \forall k>0(f(k)<H(k))\right\}$. Now a forcing notion $\mathbf{P}$ is said to have the Laver property iff for every $H \in \omega^{\omega}$ in $V$,

$$
\mathbb{1} \Vdash_{\mathbf{P}} \text { " } \forall f \in \Pi H \exists \bar{A} \in V(\bar{A} \text { is a cone covering } f) \text { ". }
$$

Like Mathias forcing, the dual-Mathias forcing has the Laver property as well and therefore adds no Cohen reals (cf. $\mathrm{GO}$ and [BJ]).

LEMMA 6.4 The forcing notion $\mathfrak{M}$ has the Laver property.

Proof: Given $f, H \in \omega^{\omega}$ such that for all $k>0: f(k)<H(k)$, let $\langle s, X\rangle$ be an $\mathfrak{M}$-condition. Because $\mathfrak{M}$ has pure decision and $f(1)<H(1)$, we find a $Y_{0} \in(s, X)^{\omega}$ such that $\left\langle s, Y_{0}\right\rangle$ decides $f(1)$. Set $s_{0}:=s$. Suppose we have constructed $s_{n} \in(\mathbb{N})$ and $Y_{n} \in(\omega)^{\omega}$ such that $s \preceq s_{n},\left|s_{n}\right|=|s|+n$ and $\left(s_{n}, Y_{n}\right)^{\omega}$ is a dual Ellentuck neighborhood. Choose $Y_{n+1} \in\left(s_{n}, Y_{n}\right)^{\omega}$ such that for all $h \in(\mathbb{N})$ with $s \preceq h \sqsubseteq s_{n}$ and $\operatorname{dom}(h)=\operatorname{dom}\left(s_{n}\right):\left\langle h, Y_{n+1}\right\rangle$ decides $f(k)$ for all $k<2^{n+1}$. Further let $s_{n+1} \in(\mathbb{N})$ be such that $s_{n} \preceq s_{n+1},\left|s_{n+1}\right|=$ $\left|s_{n}\right|+1=|s|+n+1$ and $s_{n+1} \preceq Y_{n+1}$. Finally let $Y$ be such that for all $n \in \omega$ : $s_{n} \preceq Y$. Evidently, the $\mathfrak{M}$-condition $\langle s, Y\rangle$ is stronger than the given $\mathfrak{M}$ condition $\langle s, X\rangle$ (or equal). Now if $k, n \in \omega$ such that $2^{n} \leq k<2^{n+1}$, then let $\left\{h_{j}: j \leq m\right\}$ be an enumeration of all $s \preceq h \sqsubseteq s_{n}$ with $\operatorname{dom}(h)=\operatorname{dom}\left(s_{n}\right)$. It is clear that $m<2^{2^{n}}$. Further let $A_{k}:=\left\{l \in \omega: \exists j \leq m\left(\left\langle h_{j}, Y\right\rangle \vdash_{\mathfrak{M}} f(k)=l\right)\right\}$; then $\left|A_{k}\right| \leq m<2^{2^{n}}$ and because $2^{n} \leq k$, we have $\left|A_{k}\right|<2^{k}$. If we define $A_{0}:=\{l \in \omega:\langle s, Y\rangle \Vdash \mathfrak{M} f(0)=l\}$ then the $\mathfrak{M}$-condition $\langle s, Y\rangle$ forces that $\bar{A}:=\left\langle A_{k}: k \in \omega\right\rangle$ is a cone for $f$.

Using these results we can prove the following

THEOREM 6.5 $\Sigma_{2}^{1}(\mathfrak{R})$ implies neither $\Sigma_{2}^{1}(\mathcal{L})$ nor $\Sigma_{2}^{1}(\mathcal{B})$.

Proof: Because a forcing notion with the Laver property adds no Cohen reals and because the Laver property is preserved under countable support iterations of proper forcings (with the Laver property), in the model constructed in Theorem 6.2 no real is Cohen over $L$. Therefore in this model $\Delta_{2}^{1}(\mathcal{B})$ fails and because $\Sigma_{2}^{1}(\mathcal{L})$ implies $\Sigma_{2}^{1}(\mathcal{B})$ (by [ []u] ) also $\Sigma_{2}^{1}(\mathcal{L})$ has to be wrong in this model.

REMARK: For the analogous result cf. JSS. 


\section{Appendix}

Although the Ramsey property and the dual-Ramsey property are very similar, we can show that the two Ramsey properties are different.

THEOREM 7.1 With the axiom of choice we can construct a set which is Ramsey but not dual-Ramsey.

Proof: First we construct a set $C \subseteq[\omega]^{\omega}$ which is not dual-Ramsey. The relation " $\stackrel{*}{=}$ " is an equivalence-relation on $(\omega)^{\omega}$. (Remember that $X \stackrel{*}{=} Y$ if and only if there are $f, g \in[\omega]^{<\omega}$ such that $X \sqcap\{f\} \sqsubseteq Y$ and $Y \sqcap\{g\} \sqsubseteq X$.) Now choose from each equivalence class $X^{*}$ an element $A_{X}$ and let $h_{X}:=|f|+|g|$ be of least cardinality, where $f$ and $g$ are such that $X \sqcap\{f\} \sqsubseteq A_{X}$ and $A_{X} \sqcap\{g\} \sqsubseteq$ $X$. Further define:

$$
F(X):= \begin{cases}1 & \text { if } h_{X} \text { is odd } \\ 0 & \text { otherwise. }\end{cases}
$$

Then the set $\left\{X \in(\omega)^{\omega}: F(X)=1\right\}$ is evidently not dual-Ramsey and therefore also the set $C:=\left\{x \in[\omega]^{\omega}: \exists X \in(\omega)^{\omega}(F(X)=1 \wedge x=\operatorname{pc}(X))\right\}$ is not dual-Ramsey.

Now define $r:=\{b\{k, k+1\}: k \in \omega\}$, then $\operatorname{cp}(r)=\{\omega\} \notin(\omega)^{\omega}$ and hence $[r]^{\omega} \cap C=\emptyset$. So, the set $C$ is Ramsey.

We can show that the dual-Ramsey property is stronger than the Ramsey property.

LEMMA 7.2 If $V \models \Sigma_{n}^{1}(\Re)$ then $V \models \Sigma_{n}^{1}(\mathcal{R})$.

PROOF: Given a $\Sigma_{n}^{1}$-formula $\varphi(x)$ with parameters in $V$. Let $\psi(y)$ be defined as follows.

$$
\psi(y) \text { iff } \exists x(x=\operatorname{Min}(\operatorname{cp}(y)) \wedge \varphi(x)) .
$$

We see that $\psi(y)$ is also a $\Sigma_{n}^{1}$-formula (with the same parameters as $\varphi$ ). Now if there is an $X \in(\omega)^{\omega}$ such that for all $Y \in(X)^{\omega}, \psi(\operatorname{pc}(Y))$ holds, then for all $y \in[x]^{\omega}$ where $x=\operatorname{Min}(X), \varphi(y)$ holds. The case where for all $Y \in(X)^{\omega}$, $\neg \psi(\mathrm{pc}(Y))$ holds, is similar.

With these results and all the symmetries we found between the two Ramsey properties and between the Mathias forcing and the dual-Mathias forcing, it is natural to ask whether there is a property which is equivalent to "every $\Sigma_{2}^{1}$-set of reals has the dual-Ramsey property". Another interesting open problem, which surely would give us a lot of information about the relationship between the two Ramsey properties, would be the following question:

$$
\text { Is } \Sigma_{2}^{1}(\mathcal{R}) \text { equivalent to } \Sigma_{2}^{1}(\mathfrak{R}) \text { ? }
$$

Acknowledgements: I would like to thank Pierre Matet for all the fruitful discussions concerning the results in this paper, and the referee for valuable comments on an earlier version of this paper. 


\section{References}

[BJ] T. Bartoszyński and H. Judah: "Set Theory: on the structure of the real line." A. K. Peters, Wellesley 1995.

[Br] J. BRende: Combinatorial properties of classical forcing notions. Ann. of Pure and Applied Logic 73(1995), 143-170.

[CS] T. J. Carlson and S. G. Simpson: A dual form of Ramsey's Theorem. Adv. in Math. 53(1984), 265-290.

[El] E. Ellentuck: A new proof that analytic sets are Ramsey. J. Symb. Logic 39(1974), 163-165.

[Go] M. Goldstern: Tools for your forcing construction, in "Set Theory of the Reals," (H. Judah, Ed.), pp. 305-360, Israel Mathematical Conference Proceedings, Bar-Ilan University 1993.

[Ha] L. Halbeisen: On shattering, splitting and reaping partitions, (preprint).

[HJ] L. Halbeisen and H. Judah: Mathias absoluteness and the Ramsey property. J. Symb. Logic 61(1996), 177-193.

[HM] L. Halbeisen And P. Matet, in preparation.

[Je 1] T. Jech: "Set Theory." Academic Press, London 1978.

[Je 2] T. Jech: "Multiple Forcing." Cambridge University Press, Cambridge 1987.

[Ju] H. JudAH: $\Sigma_{2}^{1}$-sets of reals. J. Symb. Logic 53(1988), 636-642.

[JS] H. Judah And S. Shelah: $\Delta_{2}^{1}$-sets of reals. Ann. of Pure and Applied Logic 42(1989), 207-223.

[Ka] I. G. Kastanas: On the Ramsey property for sets of reals. J. Symb. Logic 48(1983), 1035-1045.

[Ke] A. S. Kechris: "Classical Descriptive Set Theory." Springer-Verlag, New York 1995.

[Ku] K. Kunen: "Set Theory, an Introduction to Independence Proofs." North Holland, Amsterdam 1983.

[Mt 1] P. Matet: Partitions and filters. J. Symb. Logic 51(1986), 12-21.

[Mt 2] P. Matet: Happy families and completely Ramsey sets. Arch. Math. Logic 32(1993), 151-171.

[Mt 3] P. Matet: Combinatorics and forcing with distributive ideals, (to appear in Ann. of Pure and Applied Logic). 
[Ma] A. R. D. Mathias: Happy families. Ann. Math. Logic 12(1977), 59-111.

[Pl] S. PlewIK: On completely Ramsey sets. Fund. Math. 127(1986), 127132.

[SS] S. Shelah and O. Spinas: The distributivity numbers of products of $\mathcal{P}(\omega) /$ fin I, (preprint).

[Si] J. Silver: Every analytic set is Ramsey. J. Symb. Logic 35(1970), 60-64.

Eidgenössische Technische Hochschule

Departement Mathematik

ETH-Zentrum

8092 Zürich

Switzerland

E-mail: halbeis@math.ethz.ch 